PAPER

\title{
LILES System: Guiding and Analyzing Cognitive Visualization in Beginning and Intermediate Kanji Learners
}

\author{
Luis INOSTROZA CUEVA $^{\dagger a)}$, Nonmember and Masao MUROTA ${ }^{\dagger b)}$, Member
}

\begin{abstract}
SUMMARY This paper provides conceptual and experimental analysis of a new approach in the study of kanji, our "Learner's Visualization (LV) Approach". In a previous study we found that the LV Approach assists beginning learners in significantly updating their personal kanji deconstruction visualization. Additionally, in another study our findings provided evidence that beginning learners also receive a significant impact in the ability to acquire vocabulary. In this study, our research problem examines how beginning and intermediate students use visualization to cognitively deconstruct (divide) kanji in different ways, and how this affects their learning progress. We analyze the cognitive differences in how kanji learners explore and deconstruct novel kanji while using the LV Approach and how these differences affect their learning process while using the LV Approach. During the learning experience, our LILES System (Learner's Introspective Latent Envisionment System), based on the LV Approach, guides learners to choose from a set of possible "kanji deconstruction layouts" (layouts showing different ways in which a given kanji can be divided). The system then assists learners in updating their "kanji deconstruction level" (the average number of parts they visualize within kanji according to their current abilities). Statistical analysis based on achieved performance was conducted. The analysis of our results proves that there are cognitive differences: beginners deconstruct kanji into more parts ("blocks") than intermediate learners do, and while both improve their kanji deconstruction scores, there is a more significant change in "kanji deconstruction level" in beginners. However, it was also found that intermediate learners benefit more in "kanji retention score" compared with beginners. Suggestions for further research are provided.

key words: kanji learning approach, learning support system, visual cognition, kanji deconstruction level, cognitive maps
\end{abstract}

\section{Introduction}

\subsection{Aim of the Study}

The aim of this study was to implement a system that makes it possible to analyze the cognitive complexities kanji students face while visually dividing or "deconstructing" novel kanji during the learning process. This system, the Learner's Introspective Latent Envisionment System (LILES system), is based on our new approach called the Learner's Visualization (LV) Approach. This research is original in that it analyzes the cognitive difference between beginning and intermediate learners in the course of progressively learning novel kanji (and updating how many "blocks" or parts they visualize in each novel kanji). Following the findings that show that beginning learners tend to progress from visualiz-

Manuscript received October 20, 2010.

Manuscript revised February 7, 2011.

${ }^{\dagger}$ The authors are with the Tokyo Institute of Technology, Tokyo, 152-8552 Japan.

a) E-mail: luisu.inostroza.com@gmai.com

b)E-mail: murota@hum.titech.ac.jp

DOI: 10.1587/transinf.E94.D.1449 ing small blocks within each kanji to visualizing a few larger blocks (although they do not necessarily aim to ultimately visualize the entire kanji as just one recognizable block) [1], this study strives to determine the cognitive differences that may exist in the way beginning and intermediate learners visually deconstruct kanji, especially while learning novel kanji using the LV approach. In this paper, we discuss our experimental results and summarize the main contributions this study makes to the field of kanji learning.

\subsection{Motivation}

Although another of our previous studies showed that $b e$ ginning students of kanji receive a significant impact not only in their ability to visually deconstruct kanji but also in the ability to acquire vocabulary after studying with a Learner's Visualization (LV) Approach-based learning support system [2], it remains to be determined whether or not intermediate level students of kanji also receive a significant impact in their "kanji deconstruction level" and "kanji retention" scores. Although we could expect that any learner would gain similar benefits regardless of the learner's level, we know that only established and accepted evidence provided by research can confirm presumed assumptions. We are further motivated by the crucial need to determine not only what specific factors account for better learning results, but also how this happens. Doing so, we could certainly establish how to enhance future kanji learning systems with cutting-edge functionalities in accord with the most recent findings.

\section{Kanji Background}

\subsection{Kanji Writing System Research}

The Japanese writing system is comprised of a unique blend of scripts: kanji (which are derived from Chinese characters), two kana syllabaries (which are phonetic), Roman letters and Arabic numerals. This offers fascinating questions for research, and kanji has been the main topic in numerous studies. In one work related to the present study, Tollini remarked on the importance of understanding how Westerners "see" kanji, especially during the initial stage of the learning process [3]. He stated that Westerners recognize kanji based on visual recognition laws different than those employed by the Japanese [3]. According to the work of Takagi, the complexity of recognizing a kanji can be reduced if learners vi- 
sually break down the kanji into smaller parts, and organize them [4].

\subsection{Kanji Learning Approaches}

\subsubsection{Radical Approach}

This method uses "radicals" (in Japanese, 部首 bushu) as the key elements of any kanji, grouping kanji according to radicals present within the characters. Limitations of this approach lie in the heterogeneity of the different groupings and in the fact that there are variations on the radicals' shapes.

\subsubsection{Frequency-Based Learning Approach}

This approach is based on Monbusho's Educational Kanji Chart (教育漢字の学年別漢字配当表). The main drawback of this approach is the fact that some kanji are taught without taking into account their visual components. For example, "eat" (ta-beru, ku-rau/SHOKU) (食) is taught in the second grade, but its component, "good"(yo-i, RYOU) (良) is taught in the fourth grade. Teaching a kanji without previously teaching its elementary parts is common but may not be efficient for foreigners.

\subsubsection{Component Approach}

This approach emphasizes learning the building blocks of kanji. All the parts of a kanji are identified as components. A component is a set of strokes which keeps the same visual shape. For example, 歌 is composed of the components: 可 and 欠; another example, 東: “木 and 日” or “田 and 木”. Components are associated with a pattern, which defines the relative position of them.

\subsubsection{Learner's Visualization Approach}

This novel approach proposed by Inostroza et al involves three elements: 1. the learner's visual cognition, 2. multiform "deconstruction" of kanji, and, 3. block analysis/synthesis [1]. The first element, the learner's visual cognition, is the way in which a learner visually divides a kanji into parts or "blocks". The second element is the set of all the possible options of how that kanji could be divided or "deconstructed" into blocks, from the highest number of small blocks down to just one block (which would be the entire kanji itself as a single block). The third element is the analysis/synthesis cognitive strategy embedded in the approach which allows the user to analyze the given kanji by selecting any block inside it for further study, or to synthesize that block with a bordering block inside the same kanji, suggesting a larger block to be considered that combines the two smaller ones.

\section{Cognition Background}

\subsection{Cognitive Processing of Kanji vs. Kana}

Neuropsychological research has shown that when one reads Japanese, the kana and kanji scripts are processed by different areas of the brain. Usui et al found that kana and kanji are both processed in the LBTA (the left basal temporal area, also called the left inferior posterior temporal lobe), but, the two scripts are processed in different areas of the LBTA [5]. Interestingly, kanji is processed in the same area of the LBTA that is responsible for recognizing and naming pictures and objects. This suggests that the visual shapes of a kanji play a key role in the reader's cognitive processing, which is congruent with neuropsychological literature [6]. Nakamura et al found increased activity in this area of the LBTA during the acts of visually fixating on kanji, physically writing kanji, mentally writing kanji, and mentally recalling kanji words [7]. Ino et al concluded that the brain processes kana and kanji in different areas of the LBTA because of the quite different characteristics of the two scripts and the mental tasks required to read them [8].

\subsection{Cognitive Maps and Kanji Visualization Layouts}

Cognitive maps are a method our brain uses to accumulate and store knowledge (primarily spatial information) in the so-called "mind's eye". The brain links related visual concepts in a mental network (a "map") to facilitate their accessibility. This reduces cognitive load, and thus enhances recall and learning.

\section{Research Question and LILES System}

\subsection{Research Question}

This study is primarily focused on identifying whether or not there is a cognitive difference in beginners and intermediate learners as to their "kanji deconstruction visualization" (meaning the number of different possible deconstruction layouts learners' view per kanji) and "kanji meaning retention".

\subsection{Existing Kanji Learning Systems vs. the LILES Sys- tem}

To this day, there are no kanji learning systems currently in use that facilitate the analysis of how learners update their kanji deconstruction level, which is the purpose of this study. With the exception of the LV System that we have developed, no existing system is based on the Learner's Visualization (LV) Approach, which guides learners to explore and update the way they deconstruct (visually divide) kanji [1]. Therefore, to pursue our study we have adapted our previously implemented Learner's Visualization (LV) 
System in a way that would allow us to analyze learners' behavioral results, with the goal of eventually explaining how the LV Approach facilitates learning. We have named this system the LILES System, where LILES stands for Learner's Introspective Latent Envisionment System, to convey the concept that when learners visually deconstruct kanji into layouts of blocks (groups of strokes) and update their preferred deconstruction layout via this system, learners envision (see or picture in their mind) possible deconstruction layouts in an introspective way (given to examining one's own perceptual experience), bringing to fruition new ways to visualize each kanji that are latent in every learner's mind.

\section{LILES System Implementation}

We have implemented the LILES system as an adaptation of our LV System, which is based on our LV Approach to kanji learning. A brief functional description of the LILES System is as follows: While learning kanji using the LILES System, it encourages learners to explore a predefined set of "deconstruction layouts"for each kanji, and in doing so it guides learners in visualizing alternate deconstruction layouts which differ from the layout the learner initially preferred to visualize. The learning process, illustrated in Fig. 1, can be summarized as follows: (boxes "a", "b", "c" etc, refer to the boxes attached to the figures):

The system shows a certain kanji with its corresponding meaning, (boxes "a" and "c"), for example, the kanji for "to be surprised, to be astonished" = "odoroku" (驚). Below the kanji, the system shows a set of predefined possible deconstruction layouts into which the kanji can be divided. The user first selects the deconstruction that best matches the way he would naturally divide that kanji according to his own personal visual cognition. After selecting his deconstruction of choice, the system then allows the user to examine and learn more by clicking on the zone of particu-

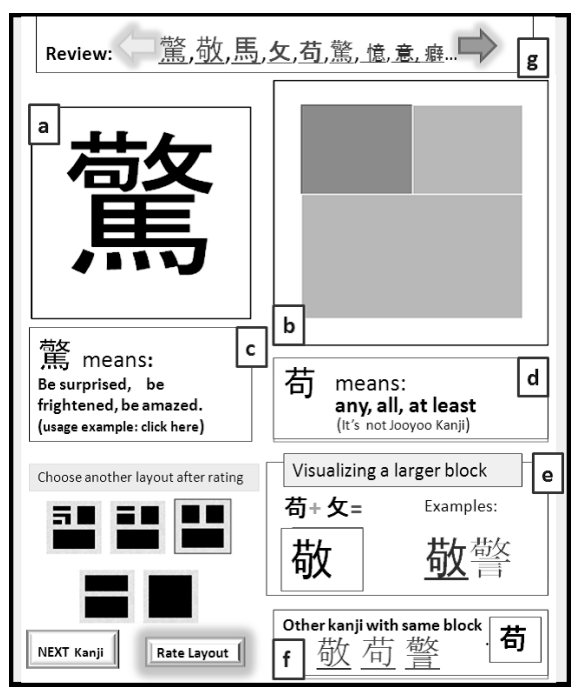

Fig. 1 Functionality of the LILES system. lar blocks, (box "b"), within his chosen deconstructions as well as blocks within all the other possible deconstructions. When a user clicks on the zone of a block, (box "b"), the system gives relevant information about it, (box "d"). The system then suggests that the learner consider a new, larger and more complex block, (box "e"), which is constructed based on the block selected by the learner combined with an additional block also present in the same kanji, bordering the selected block. The new assembled block becomes a"superblock"in the kanji in study. While the user is working on the kanji 驚 and examining the block 苟, the system suggests the construction of a new block “敬”, combining (苟) with adjacent block (父), and shows some examples of other kanji that include this more complex superblock. Learners can explore any of the examples just by clicking on one of them. The core of the technique lies with the recommendation of a "next complex block". For example, if the learner selected the block “苟', the “next complex block” suggested by the system is “苟+父” = “敬”. Alternatively, at the bottom of the learning environment users can choose to explore other kanji that include the block they selected, (box "f"). The kanji the user has studied kanji appear at the top of the screen in case she wishes to review them, (box "g").

\section{Experimental Analysis with the LILES System}

Experimental analyses were conducted via our LILES System to obtain necessary values for analyzing the possible cognitive difference in kanji deconstruction level after using the LV approach-based learning support system. A brief description of the experiments is as follows.

\subsection{Evaluation Questions}

Question one: "Do beginning and intermediate learners deconstruct kanji differently?" "Does the LV Approach assist both beginning and intermediate learners equally in updating their personal way of visualizing kanji?". Question two: "Do both groups receive an equally significant benefit in their "kanji retention score" (vocabulary) while learning via the LV approach?"

\subsection{Evaluation Method}

For the learning session learners used our LILES System, an implemented web-based system which is an improved version of our Learner's Visualization (LV) System. Learners were randomly assigned to one of two variants: the Control Group (G1), which used the kanji-learning software with only one possible deconstruction layout offered per kanji, and the Experiment Group (G2), which used the same software but with a function that allows the learner to choose her deconstruction layout of preference from a group of predefined possible layouts for each kanji.

\subsection{Participants}

A total of 96 university students of 21 different nationalities 
were evaluated and classified as "beginning level learners" (48 students) and "intermediate level learners" (48 students) following the criteria of the vocabulary knowledge scale developed by Paribakht and Wesche [9]. The mean length of formal study (i.e. under a program with a plan, a goal and a way to measure progress) for beginning students was 137 hours, and 371 hours for intermediate students. The participants were exclusively speakers of languages that use an alphabetic script.

\subsection{Instructional Materials}

$\square$ Global selection. The kanji database obtained from the Asahi Newspaper published between 1985 and 1998 serves as the basis for the global selection: a total of 6,355 kanji out of almost 49,000 kanji included in the 13-volume Morohashi Dai Kanwa Jiten. The distribution of this database (being $X=$ number of strokes) was obtained in Tamaoka's study. Its negative hypergeometric distribution has as parameters: $K=24.6877, M=6.8335, n=41, D F=26,(X)^{2}=$ 45.63, $P=0.01, C=0.0072[10]$.

$\square$ Specific selection. The specific selection criterion was visual complexity which was found strongly positive correlated with the number of strokes [11]. This criterion was chosen based on the importance of form, stressed by Tollini [3], in the selection of kanji to be taught. The rating data was based on the negative hypergeometric distribution of the 6,355 kanji. From this database only a set of 200 were selected ( 73 percent belonging to the $j \bar{o} y \bar{o}$ kanji list issued on October 10, 1981, and the remaining 54 kanji were the most common non-jōyo kanji that are either: (a) useful as building-blocks for other kanji (e.g. 吾, 勿, 頁, etc) or (b) very common in names (e.g. 藤, 岡, etc)). The sampling kanji were randomly selected proportionally to the distribution of the database, i.e., frequency of each value (number of strokes). This set of 200 kanji is called in this study: "databasekanji" $=d_{k}$.

$\square$ Kanji deconstruction and deconstruction layouts. Nara's study previously established a tentative set of kanji parts that non-native students see in kanji [12]. However, as different foreigners may have different ways of deconstructing kanji, in order to have a more comprehensive range of possible deconstruction layout options, a preliminary experiment was conducted. The goal was to obtain an experimental and open-to-consideration set of those kanji deconstruction layouts that are visually salient to non-native learners. This experimental set has been elaborated based on the survey done in Nara's study on kanji visual salient parts [12]. Compared with Nara's survey which had only four participants, our survey was conducted with seven non-native speakers of Japanese enrolled in the University of Tokushima's Japanese language course (3 intermediate learners and 4 beginning learners). They were given 200 randomly selected jōyo kanji and were asked to write down all the possible deconstruction layouts they visualize in each kanji that make sense according to their personal visual cognition, indicating at the end their preferred layout. The results showed that beginners tend to visualize substructures within a given kanji in a much different way than intermediate learners do. The majority of the time, participants preferred to deconstruct the given kanji into 2 or 5 blocks (Intermediate students: mean=2.93, SD 'standard deviation' $=0.74$; Beginning students: mean $=3.95$, $\mathrm{SD}=1.17$; Global: mean=3.44, $\mathrm{SD}=1.098$ ). The number of deconstruction layouts created per kanji mainly varied between 4 to 6 different layouts (mean=4.739, $\mathrm{SD}=1.322$ ).

$\square$ Unknown kanji selection per groups. As the study examines the ability to learn and visualize novel kanji, the characters used in the target items were chosen kanji that are typically not well known. In order to confirm selection of unknown kanji to be used in the learning session of the experiment, a kanji test was given to all participants: $K R T($ Kanji Retention Test $)=$ Pre-test for experiment 2. See details in Sects. 6.6 and 6.7.

$\square$ Time for learning selected kanji. All participants in both groups were instructed to learn the list of 100 kanji in 60 minutes.

\subsection{Design}

Two experiments were conducted:

1. Experiment 1: evaluates the comparison of the means of kanji deconstruction level between beginning and intermediate learners.

2. Experiment 2: evaluates the comparison of the means of kanji retention score between same groups.

The study design was conducted as follows:

- Pre-tests:

1. Pre-test for Experiment 2: 50 minutes for checking vocabulary of $d_{k}$ (database danji $_{\text {of }} 200$ kanji) and building a database of 100 novel kanji.

2. Pre-test for Experiment 1: 25 minutes for selecting layouts for each of the 100 novel kanji. (followed by a 7-minute break)

\section{- Learning session:}

3. Group 1: 60 minutes exploring one layout per kanji (100 novel kanji in total)

4. Group 2: 60 minutes exploring the different deconstructions layouts of the 100 novel kanji. (a 7-minute break)

\section{- Post-tests:}

5. Post-test for Experiment 1: 25 minutes for choosing the participant's final preferred visual deconstruction layout for each of the 100 novel kanji.

6. Post-test for Experiment 2: 60 minutes for checking vocabulary of each of the 200 kanji of database $d_{k}$.

The design of the two experiments was as follows:

$\star$ Type of experiment: Research Experiment, $2 \times 2 \times 2$ ANOVA 
Design. Three-way ANOVA with one RM (repeated measure). The RM factor has 2 levels.

$\star$ Dependent variable: For Experiment 1, "Kanji Visualization Score" (KV Score), or "kanji visual deconstruction score", the mean number of blocks into which the user chooses to deconstruct given kanji). For Experiment 2, "Kanji Retention Score" (KR Score), the mean retention of given kanji's meaning.

$\star$ Independent variables (factors): The two levels (Pre-Post) of the RM (within-group) factor called Tests are measurements taken before and after treatment. The two betweengroup factors were Groups (Control Group or Experimental Group) and, Learners (Beginning or Intermediate). The levels of the Groups factors are defined by the presence or absence of the "Kanji Deconstruction Layout Exploration" function in the learning support system, which allows the user to explore and study multiple deconstruction layouts for each featured kanji (this function is the characteristic feature of the LV Approach).

$\star$ Goal: Compare the performance achievements between beginning and intermediate learners.

\subsection{Instruments}

$\nabla$ Kanji Visualization Test $(K V T)$. For Experiment 1, a test with maximum score 1.00 was designed to measure the dependent variable, kanji visual deconstruction level, referred to as Kanji Visualization Score ( $K V$ Score).

$\nabla C a l c u l a t i o n$ method of KVT. The dependent variable was calculated based on the mean of the normalization of the number of blocks mentally visualized per kanji. We used the formula score $_{k}=(B-n+1) / B$ where score $_{k}$ represents the score in a given kanji, $B$ represents the maximum number of blocks in that kanji, and $\mathrm{n}$ expresses the number of blocks mentally visualized per kanji. Consequently to visualize a kanji as a whole unit scores 1 point, and the mean of all the scores $_{k}$ makes the kanji deconstruction level in a learner.

$\triangle$ Kanji Retention Test $(K R T)$. For Experiment 2, a kanji vocabulary test was conducted as a Pre-test and Post-test. The test was designed to measure the dependent variable, retention of meaning. The dependent variable was calculated based on the score on the kanji tests, referred to as Kanji Retention Score (KR Score). The instructions implied that participants needed to consider how well they actually knew the kanji so that our result would reflect only truly assimilated knowledge of the kanji as opposed to vague knowledge. This criterion was included in order to correctly measure only the appropriate depth of vocabulary knowledge as defined by Paribakht [9].

$\triangle$ Calculation method of KRT. Procedure and calculation method of kanji retention test were as follows. Learners were presented with a list of target kanji and asked to choose their level of knowledge for each kanji according to the following self-reporting categories based on an adaptation of the Vocabulary Knowledge Scale (VKS) [9]:

I I do not remember having seen this kanji before.

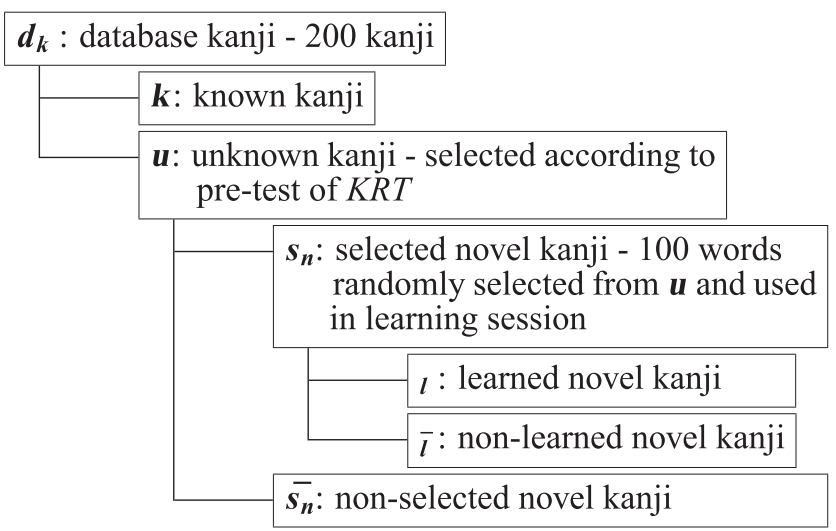

Fig. 2 Database for the pre-tests and post-tests.

II I am sure I have seen this kanji before, but I am also sure that I do not know what it means.

III I think I know this kanji. I have seen it before, and I think it means _ . _ _ _ _..(translation or synonym)

IV I know this kanji. It means _. . . . ...(translation or synonym)

V I can use this kanji in context: _. . . . . ...(Write an example). (And please also do section IV)

Total score is calculated based on: Categories I and II represent score of 0.0 and 0.05 respectively. Wrong responses in levels III, IV or V lead to a score of 0.05. A score of 0.1 is given if there is a good response in category III, and 0.2 in category IV. For category V, a score of 0.3 is given if the kanji is used in the correct context but with incorrect usage and no translation is provided (and 0.4 when translation is provided). A score of 0.5 is given when translation was provided and the kanji has been used in the right context and with accurate usage, even if other parts of the sentence may have errors. As the number of kanji included in the pretests and post-tests of Experiment 2 was 200, the maximum score was 100 . It is worth mentioning that although in the learning session participants learn only novel kanji, in the pre-test and post-test of Experiment 2 they encounter the same set of kanji called "database" $=d_{k}$. Figure 2 illustrates the kanji database description for both experiments.

\subsection{Instructions and Procedure}

The sequence of instructions for the participants was:

- Pre-tests:

1. KRT=Pre-test for Experiment 2: All participants received 200 characters $=d_{k}$, and they were asked to answer the KRT Questionnaire (explained in Sect. 6.6). Participants were instructed to check appropriately instead of guessing about their knowledge of kanji. When they checked either (I) or (II), the respective kanji were selected for the experiment, generating a pool of novel kanji for the experiment $=s_{n}$. 
Learners were given 50 minutes for the test. In this experiment, the resulting pool of novel kanji was always over 100 kanji.

2. $K V T=$ Pre-test for Experiment 1: Learners were given 25 minutes to choose their initial preferred deconstruction layout from a predefined list of possible deconstruction layouts for each of 100 novel kanji. The kanji used were randomly selected from the pool of novel kanji.

- Learning session:

3. Control Group: Learners were given 60 minutes to learn the set of 100 novel kanji without having the option to explore alternate deconstruction layouts for each given kanji. They were instructed to study each kanji by exploring the blocks (parts) of the sole layout that was displayed beside each kanji. The system allowed learners to continue on to study the next kanji only after exploring at least half of the blocks of the given kanji layout. The solitary layouts were exactly the ones learners chose as their preferred layouts for each kanji from the list of 100 novel ones (i.e. during step two: $K V T=$ Pre-test for Experiment 1)

4. Experimental Group: Learners were given 60 minutes to learn the set of 100 novel kanji from the LILES system with the option to explore alternate deconstruction layouts per kanji.

- Special instruction for Experimental Group: In order to ensure that learners explored the alternate deconstruction layouts for each kanji, they were asked to rate the alternative layouts according to their perceived usefulness for generating semantic clues for memorizing meaning of the kanji as a whole. They were asked to pay close attention to all options of semantic links that could be activated before rating. The rating option was able only available after some exploration of at least half of the blocks per layout. Learners were also instructed to complete the explorations at their own pace and spend as much time as they needed even if they could not complete the task of learning the entire set of given kanji. Although all study participants were able to see kanji information on each kanji as soon as the given kanji appeared, Control Group learners were not able to explore alternate deconstruction layouts beyond their initial chosen layout for that kanji.

\section{- Post-tests:}

5. Post-test for Experiment 1: Learners were given $25 \mathrm{~min}-$ utes to choose their final preferred visual deconstruction layout from a pre-defined list of possible layouts for each of the 100 kanji in study.

6. Post-test for Experiment 2: Learners were given $60 \mathrm{~min}-$ utes for the Kanji Retention Test containing the same set of kanji as in pre-test $=d_{k}$.

There was a 7-minute break between tests and learning session. To deter students from reviewing kanji during the break periods, they were asked to briefly share with one another the most unforgettable experience of their life.

\section{Results}

All statistical analyses were performed using the PASW (Predictive Analytics SoftWare) Statistics 18 software version (the SPSS version between 2009 and 2010). The descriptive statistics results are presented in Table 1, and a comparison of the improvements of KV Score and KR Score, by Learner and by Group, between pre-test and post-test is summarized in Table 2. Analysis of variance (ANOVA) was used to assess the changes in KV Score and KR Score. However, prior to the ANOVA test, Levene's Test for Equality of Variances was performed in order to assess the assumption that the population variances are equal. The Levene's test was negative, i.e., not positive $(P>0.05): P>0.589$ for data for Experiment 1, and $P>0.195$ for Experiment 2, meaning the variances in the groups were equal (homogeneous, i.e. homogeneity of variance).

The results of the ANOVA are summarized in Table 3 and Table 4. It was found that there is an interaction between all factors. This three-way interaction shows that two-way interactions vary across levels of the third variable. Additionally, since all of the factors in this three-way ANOVA are dichotomous ( 2 levels), there was no follow-up tests to perform on significant main effects.

Table 1 Data summary of experiment 1 and experiment 2.

\begin{tabular}{|c|c|c|c|c|c|c|c|}
\hline \multicolumn{3}{|c|}{ Factors } & & \multicolumn{2}{|c|}{ Exp. 1 (KV Score) } & \multicolumn{2}{|c|}{ Exp. 2 (KR Score) } \\
\hline Learner & Test & Group & $N$ & Mean & SD & Mean & SD \\
\hline \multirow[t]{4}{*}{ Beginning } & \multirow[t]{2}{*}{ Pre } & Control & 24 & .3742 & .0279 & 10.42 & 2.796 \\
\hline & & Exp. & 24 & .3721 & .0188 & 10.21 & 1.888 \\
\hline & \multirow[t]{2}{*}{ Post } & Control & 24 & .4033 & .2745 & 19.83 & 2.713 \\
\hline & & Exp. & 24 & .5617 & .0193 & 31.25 & 2.327 \\
\hline \multirow[t]{4}{*}{ Intermediate } & \multirow[t]{2}{*}{ Pre } & Control & 24 & .6271 & .0249 & 35.71 & 2.493 \\
\hline & & Exp. & 24 & .6171 & .0271 & 34.71 & 2.710 \\
\hline & \multirow{2}{*}{ Post } & Control & 24 & .6562 & .0245 & 54.33 & 3.239 \\
\hline & & Exp. & 24 & .7258 & .0273 & 71.63 & 3.716 \\
\hline
\end{tabular}

Table 2 Difference of pre- and post-tests ( $\triangle K V$ Score and $2 \triangle K R$ Score).

\begin{tabular}{|l|r|r|r|}
\hline Factors & Exp. 1 $(K V$ Score $)$ & Exp. 2 (KR Score $)$ \\
\hline Learner & Group & $\triangle K V$ Score & $\Delta$ KR Score \\
\hline \multirow{2}{*}{ Beginning } & Control & .0291 & 9.41 \\
\cline { 2 - 4 } & Exp. & .1896 & 21.04 \\
\hline Intermediate & Control & .0291 & 18.62 \\
\cline { 2 - 4 } & Exp. & .1087 & 36.92 \\
\hline Exp.1: Experiment $1, \triangle K V$ Score : Improvement $K V$ Score \\
\hline
\end{tabular}

Table 3 Data summary of ANOVA for experiment 1.

\begin{tabular}{|l|r|r|r|r|r|}
\hline Dependent Variable: Kanji Visualization Score \\
\hline Source & Type III SS & df & Mean Square & F & Sig. \\
\hline Corrected Model & $3.273 \mathrm{a}$ & 7 & .468 & 753.488 & $<0.001$ \\
\hline Intercept & 56.442 & 1 & 56.442 & 90952.466 & $<0.001$ \\
\hline Learner & 2.512 & 1 & 2.512 & 4047.423 & $<0.001$ \\
\hline Test & .382 & 1 & .382 & 614.981 & $<0.001$ \\
\hline Group & .140 & 1 & .140 & 225.203 & $<0.001$ \\
\hline Learner*Test & .020 & 1 & .020 & 31.588 & $<0.001$ \\
\hline Learner*Group & .028 & 1 & .028 & 45.174 & $<0.001$ \\
\hline Test*Group & .173 & 1 & .173 & 278.457 & $<0.001$ \\
\hline Learner*Test*Group & .020 & 1 & .020 & 31.588 & $<0.001$ \\
\hline Error & .114 & 184 & .001 & & \\
\hline Total & 59.829 & 192 & & & \\
\hline Corrected Total & 3.387 & 191 & & & \\
\hline a. R Squared= .966 (Adjusted R Squared & \multicolumn{7}{l}{} \\
\hline
\end{tabular}


Table 4 Data summary of ANOVA for experiment 2.

\begin{tabular}{|l|r|r|r|r|r|}
\hline Dependent Variable: Kanji Retention Score \\
\hline Source & Type III SS & df & Mean Square & F & Sig. \\
\hline Corrected Model & $75865.479 \mathrm{a}$ & 7 & 10837.93 & 1397.952 & $<0.001$ \\
\hline Intercept & 215606.021 & 1 & 215606.0 & 27810.381 & $<0.001$ \\
\hline Learner & 46625.333 & 1 & 46625.33 & 6014.063 & $<0.001$ \\
\hline Test & 22188.000 & 1 & 22188.00 & 2861.964 & $<0.001$ \\
\hline Group & 2268.750 & 1 & 2268.750 & 292.639 & $<0.001$ \\
\hline Learner*Test & 1887.521 & 1 & 1887.521 & 243.466 & $<0.001$ \\
\hline Learner*Group & 77.521 & 1 & 77.521 & 9.999 & $<0.003$ \\
\hline Test*Group & 2685.021 & 1 & 2685.021 & 346.333 & $<0.001$ \\
\hline Learner*Test*Group & 133.333 & 1 & 133.333 & 17.198 & $<0.001$ \\
\hline Error & 1426.500 & 184 & 7.753 & & \\
\hline Total & 292898.000 & 192 & & & \\
\hline Corrected Total & 77291.979 & 191 & & & \\
\hline a. R Squared= .982 (Adjusted R Squared $=.981)$ & & \\
\hline
\end{tabular}

\subsection{Kanji Visualization Score}

The dependence of $K V$ Score differences between beginning and intermediate learners on Group factor (Control Group/Experimental Group) and Test factor (Pre-test/Protest) was reflected in a significant Learner $\mathrm{x}$ Group $\mathrm{x}$ Test interaction in an overall ANOVA of these data, $F(1,184)=$ $31.588, p<0.001$. Our results further indicate that this three-way interaction was due in part to the presence of a significant Learner $\mathrm{x}$ Group interaction, $F(1,184)=45.174$, $p<0.001$. The results are significant at the 0.1 percent of significance level. Therefore, one would reject the null hypothesis, concluding that there is strong evidence that the expected values in the groups differ.

\subsection{Kanji Retention Score}

The dependence of KR Score differences between beginner and intermediate learners on treatment and test was also reflected in a significant Learner $x$ Group $x$ Test interaction in an overall ANOVA of these data, $F(1,184)=17.198$, $p<0.001$. Moreover, it was found that all the two interactions add to the overall three-way interaction: a significant Learner x Group interaction $(F(1,184)=9.999, p<0.003)$, and a Learner $\mathrm{x}$ Test interaction $(F(1,184)=243.466, p<$ $0.001)$, as well as a Test $\mathrm{x}$ Group interaction $(F(1,184)=$ $346.333, p<0.001)$.

\subsection{Graphical Explanation of Results}

A graphical explanation of these results is as follows:

$\star$ Figures $3 \& 4$ illustrate the results of our study as to three factors: Test (Pre-test and Post-test), Group (Control Group and Experimental Group) and Learner (Beginning and Intermediate level).

$\star$ In Fig. 3, the learner's Kanji Visualization Score ( $K V$ Score) is shown on the y-axis. The Test factor (Pre-test vs. Post-test) is shown on the $\mathrm{x}$-axis.

$\star$ In Fig. 4, the learners' Kanji Retention Score (KR Score) is shown on the $\mathrm{y}$-axis. The Test factor is shown on the $\mathrm{x}$ axis.

$\star$ In both Figs. 3 \& 4: Results for learners in the

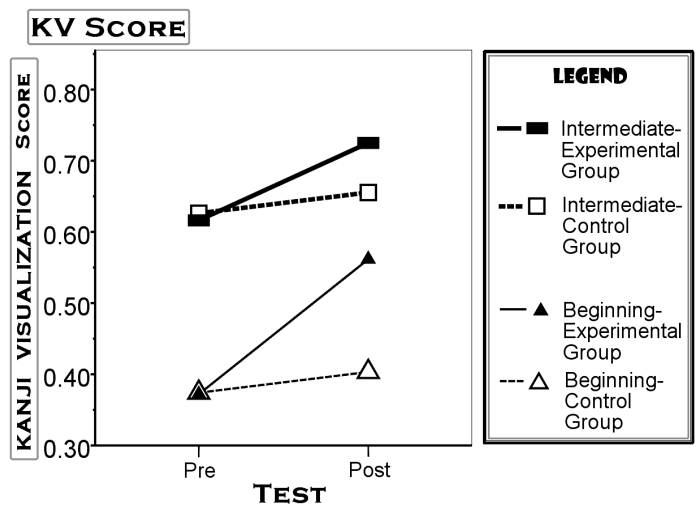

Fig. 3 Experiment 1: Comparison of $K V$ Scores obtained in pre-tests and post-tests, by learner and by group factors.

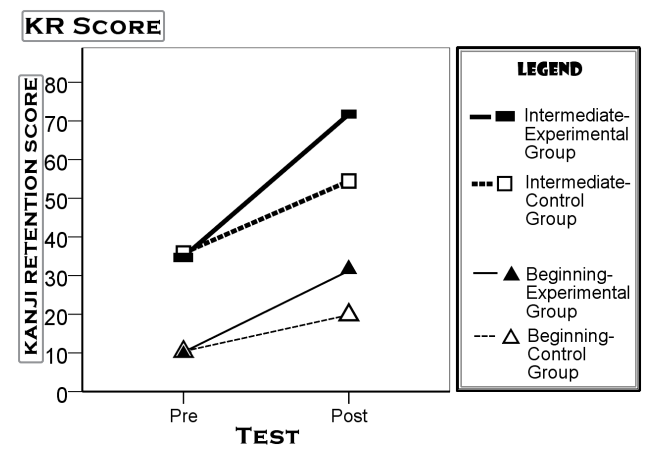

Fig. 4 Experiment 2: Comparison of KR Scores obtained in pre-tests and post-tests, by learner and by group factors.

Beginning-Control and Beginning-Experimental Groups are represented by triangles ( $\Delta$ and $\boldsymbol{\Delta}$ ) respectively. Results for learners in the Intermediate-Control and IntermediateExperimental groups are represented by squares ( $\square$ and $\boldsymbol{\square}$ ) respectively.

$\star$ The change between Pre-test and Post-test in $K V$ Score and $K R$ Score for the Control Groups is illustrated with dashed lines (- - ). The change in KV Score and KR Score for the Experimental Groups is illustrated with solid lines (-). Thin lines are used to illustrate the change for the Beginning Groups and thick lines are used to illustrate the change for the Intermediate Groups.

- Figure 3: Kanji Visualization Score

$\star$ Results of the learners' $K V$ Score summarized in Table 2 and illustrated in Fig. 3 reveal there is an appreciable difference for both beginning and intermediate learners in the Experimental Groups in how they perform between the Pre-test and Post-test. Moreover, statistical analysis indicates that beginners had a significantly larger improvement in their $K V$ Score on the Post-test when compared with intermediate learners, $(\mathrm{t}(46)=39.54, p<0.01)$.

$\star$ As beginners had a lower $K V$ Score mean to start with (Pre-test: KV Score $=0.3721$ in the Experimental Group) than intermediate learners, they had a substantially better chance of benefiting from the system (Post-test: $K V$ 
Score $=0.5617$ in the Experimental Group). Although intermediate learners had a better $K V$ Score overall (Post-test: $K V$ Score $=0.6171$ in the Experimental Group), they had an increment rate in $K V$ Score that was actually less than that of the beginners' rate $(17.61 \%$ vs. $50.95 \%)$.

$\star$ Thus, as presented in Table 2 and illustrated in Fig. 3, as to kanji deconstruction level, beginning learners gain the most benefit when studying via our Learner's Visualization Approach.

\section{- Figure 4: Kanji Retention Score}

$\star$ Results of the learners' KR Score summarized in Table 2 and illustrated in Fig. 4 reveal that there is an appreciable difference for both beginning and intermediate learners in the Experimental Groups in how they perform between the Pre-test and the Post-test. A noteworthy finding is that while beginners increased their Kanji Visualization Score much more than intermediate learners did, in contrast, it was the intermediate students who increased their Kanji Retention Score much more than beginners did $(\mathrm{t}(46)=24.238$, $p<0.01)$.

\subsection{Summary of Results}

As shown in Table 2 and illustrated in Figs. 3 \& 4, as to kanji visualization level and kanji vocabulary retention, although both the Control Groups and the Experimental Groups achieved better scores, the benefit was more significant for the Experimental Group learners who studied via our Learner's Visualization Approach. To sum up, ANOVA results of the comparison in scores for both groups of learners (i.e. beginning and intermediate learners) shows that their means are statistically different with the use of the LV Approach.

\section{Discussion}

Our results provide evidence that between beginning and intermediate learners, there are differences not only in the way they visually deconstruct kanji, but also in the degree to which the LV Approach can help them accelerate their progress in acquiring kanji vocabulary.

\subsection{Kanji Visualization Score}

Our results show that there are cognitive differences in the way beginners and intermediate learners visually deconstruct kanji: intermediate learners deconstruct each kanji into fewer individual blocks than beginners do. Besides, although there is a significant $K V$ Score ("kanji deconstruction level") improvement in both groups of learners, a statistical analysis to the improvement means shows that it is more significant in beginners.

This study began with a previous finding showing that the LV Approach assists beginning learners in accelerating their natural progression towards perceiving individual kanji in fewer numbers of blocks (as learners gradually gain the skill to recognize larger and more complex blocks within each kanji, they no longer need to "divide" that kanji into smaller blocks in order to recognize the blocks) [2]. However, this does not mean that the initial tendency of visualizing fewer blocks would reach a total holistic achievement. On the contrary, it is expected that advanced learners would eventually decrease their $K V$ Score [16]. This apparent contradiction is explained as follows:

- The results of our experiments further corroborate the conclusion of Everson and $\mathrm{Ke}$ [14] that intermediate learners tend to holistic views in character visualization. However, since in this study advanced learners were not considered, we can not corroborate Everson's additional conclusion that advanced learners eventually tend to reverse this process, going back to more analytical procedures during character visualization.

* The reason for this trend to move towards a holistic visualization and then return to a somehow more analytical visualization can be explained with the findings of Koda that suggest that what really distinguishes native from nonnative performance is the ability to detect "sub-character information validity" [15]. Moreover, congruent with Koda's conclusion, Toyoda found in a recent study that the awareness of sub-character information validity starts to develop at the advanced level, while intermediate learners still tend to overgeneralize these units [16].

$\diamond$ Additionally, although the Western perception and the Japanese perception of kanji may not differ widely from each other [13], it was reported that non-native learners of Japanese tend to ask questions on kanji substructures that native speakers do not [17]. Additionally, some studies suggest that native speakers may process kanji at the character level without relying on information from the substructures [16].

$\diamond$ The fact that the improvement in KV Score (kanji visualdeconstruction level) is stronger in beginning students than in intermediate students could be explained by the following two reasons: 1) intermediate learners have less room for improvement [number of kanji and blocks left to learn] than beginners do; 2) Intermediate students may have already developed the ability to (consciously or subconsciously) recognize blocks within individual kanji. Therefore, an LV Approach-based system can improve the intermediate students' kanji deconstruction level, but only moderately so. Their kanji visualization skills have already developed, regardless of how lengthy and arduous the learning process has been. Beginning students however have not yet developed their kanji visualization skills and thus have the most to gain from an LV Approach-based system.

\subsection{Kanji Retention Score}

A better improvement in KR Score by intermediate learners could be explained by the fact that they have already learned compositional structures of kanji, with the division of kanji into blocks, and the subsequent interpretation and assimilation of new kanji. In studying new blocks (parts) within 
kanji, intermediate learners may gain new "banks" of understanding from which they build "bridges" (connections), that according to Russel, form the kanji learner's expanding "field of confidence" for learning [13].

The improvement in kanji retention score follows the concept that the constituent parts (blocks) of kanji can be organized to improve vocabulary, taking advantage of the fact that kanji containing common substructures may share semantic roots [18]. One objection to this method may be that visual salience of opaque components (constituent parts) activates a mix of semantic representations in the Japanese lexicon, even when the salient part is not at all semantically related to the kanji as a whole, for example Flores found that the meaning of “口" (mouth) was activated when viewed within the kanji “石” (stone) [19]. However, far from being a disadvantage, foreigners use this phenomenon to build and reinforce their vocabulary through elegant (and often hilarious) mnemonic devices, e.g. "please, don't shut my mouth with a stone" or "a stone was brought and placed over the mouth of the den" [13]. Therefore, it seems that exploring deconstruction layouts results in strengthening the links between characters and their respective substructures. Finally, this suggests that the non-native learner should be encouraged to explore kanji substructures in a self-directed manner to find the kanji visual-deconstruction layout that is most meaningful for him personally. Thus the learner can discover links between the substructures of kanji that are most helpful for his personal learning experience, in conjunction with explicit guidance, and employing a wise mix of selfchallenge and received-support.

\section{Conclusion and Future Work}

In conclusion, although both beginning and intermediate learners achieve improved kanji deconstruction levels when studying via our Learner's Visualization Approach, the benefits are more significant for beginners. Further, the tendency indicating that the more possible visual deconstructions learners explore for each kanji, the more they increase their average kanji deconstruction level holds true up to the intermediate level, however, according to related scientific works, the average deconstruction level is expected to eventually decrease when going from intermediate level to advance (native-like) performance. This is due to the fact that advanced learners have developed an awareness of the validity of relevant substructures. It should be noted that the educational technique of our LILES System lies in its strategy to help learners, through exploration of deconstruction layouts, to visually deconstruct kanji in ways they have not previously experienced. In doing so, LILES appears to foster learners' cognitive processing of self-generating memory aids that mentally link substructures (blocks) and the kanji that contain them, facilitating learning progress.

\subsection{Achievements}

- First comparative analysis of the personal visual per- ception of learners when deconstructing kanji using an implemented kanji-learning educational tool: our LILES System.

- Findings that prove beginners and intermediate learners do visually deconstruct kanji differently.

- Findings that prove our LV approach can help both beginning and intermediate learners accelerate their progress in acquiring kanji vocabulary.

\subsection{Future Work}

- To evaluate the normal speed at which learners update their kanji deconstruction level and compare the rate of acceleration with the use of the LILES system for beginning, intermediate and advanced learners.

- To implement a visual aid in the LILES System that would allow learners to rate their sense of visual familiarity with each kanji, and evaluate possible correlations.

- To implement a function capable of measuring the degree of satisfaction that learners feel with each deconstruction layout while updating their kanji deconstruction visualization (the number and complexity of blocks visualized per kanji).

$\diamond$ Time passes irrevocably, as the classical Roman poet Publius Vergilius Maro said. With the passage of time, wellfounded approaches to the study of kanji mature and gain acceptance, or they give way to new ideas. First there was the Radical Approach, which came from ancient times, in which learners studied "radicals" with not rarely confusingly vague semantic values. The current kanji-learning phenomenon is in the Component Approach, which directs the learner to, passively stare at kanji, waiting to recognize the shapes she is expected to see, all while she is perfectly capable of recognizing and synthesizing shapes within that kanji, if only her personal visual cognition could be allowed to work with the shapes she herself naturally recognizes. Now we propose the Learners' Visualization Approach (or LV Approach, for short), which finally allows the learner to take the reins as she selects the layout of visual shapes closest to that she herself recognizes within a particular kanji, and gradually analyzes, defines, and synthesizes these shapes ("visuospatial temporal blocks") at her own pace and according to her own visual cognition. Though it has only just been recently proposed, we believe the Learner's Visualization Approach has the potential to become the third wave in the study of kanji. [LIC]

\section{Acknowledgments}

This study was supported by a Grant from the Ministry of Education of the Japanese Government, the Monbukagakusho Scholarship, to the first author.

The authors express their gratitude to Ms. Kathy Rice for her priceless suggestions and comments, as well as her expertise and assistance while working together with the 
first author in checking this work and proofreading.

\section{References}

[1] L. Inostroza Cueva, et al., "Cognitive effects of the LV approach on Kanji learners: A novel approach using learner's personal visual cognition," 39th ASEE/IEEE Frontiers in Education Conference, pp.M3J-1-M3J-6, San Antonio, Texas, Oct. 2009.

[2] L. Inostroza Cueva, et al. "The LV approach, a revolutionary approach to the study of Kanji: First use of the learner's visual cognition in a Kanji-learning support system," Educational Technology Research, vol.33, pp.1-10, 2010.

[3] A. Tollini, "The importance of form in the teaching of Kanji," 世 界の日本語教育 (Sekai no Nihongo Kyooiku), vol.4, pp.107-116, June 1994.

[4] H. Takagi, "Study of Kanji pattern recognition and Kanji acquisition among non-kanji area students (in Japanese)," Japanese language education around the globe, vol.5, pp.125-138, 1995.

[5] K. Usui, et al., "Processing of Japanese morphogram and syllabogram in the left basal temporal area: Electrical cortical stimulation studies," Cognitive Brain Research, vol.24, pp.274-283, 2005.

[6] I. Law, "Human brain mapping under increasing cognitive complexity using regional cerebral blood flow measurements and positron emission tomography," Danish Medical Bulletin, vol.54, pp.289305, Nov. 2007.

[7] Nakamura et al., "Modulation of the visual word retrieval system in writing: a functional MRI study on the Japanese Orthographies," Journal of Cognitive Neuroscience, vol.14, pp.104-115, 2002.

[8] T. Ino, et al., "Recognition and reading aloud of kana and kanji word: An fMRI study," Brain Research Bulletin, vol.78(2009), pp.232239, Dec. 2008.

[9] T. Paribakht and M. Wesche, "Reading comprehension and second language development in a comprehesion-based ESL program," TESL Canada Journal, vol.11, no.1, pp.9-29, 1993.

[10] K. Tamaoka and G. Altmann, "Mathematical modelling for Japanese Kanji strokes in relation to frequency, asymmetry and readings," Glottometrics, vol.10, pp.16-19, 2005.

[11] H. Kaiho and Y. Inukai, "An analysis of Gestalt characteristics of 881 Japanese/Chanese kanji," Japanese Journal of Psychology, vol.53, pp.312-315, 1982.

[12] H. Nara, "Visual salience as a search category in a kanji dictionary in interactive Japanese: Understanding written Japanese," System, vol.20, no.1, pp.75-91, 1992.

[13] B. Russel, "Kanji no Satori," The University of British Columbia, pp.1-110, April 1995.

[14] M. Everson and C. Ke, "An inquiry into the reading the reading strategies of intermediate and advanced learners of Chinese as a foreign language," Journal of of the Chinese Language Teacher Association, vol.32, pp.1-20, 1997.

[15] K. Koda, "Development of L2 intraword orthographic sensitivity and decoding skills," The Modern Language Journal, vol.83, no.1, pp.51-64, 1999.

[16] E. Toyoda,“An analysis of L2 readers' commnets on Kanji recognition," Electronic Journal of Foreign Language Teaching, vol.6, no.1, pp.5-20, 2009.

[17] H. Abramson, et al., "The logic of kanji lookup in a JapaneseEnglish Hyperdictionary," ALLC-ACH, Bergen, Norway, June, 1996.

[18] H. Abramson, S. Bhalla, K. Christianson, J. Goodwin, J. Goodwin, J. Sarraille, and L. Schmitt, "Recognition of Japanese characters by non-native learners through a support database system," Proc. Second International Conference on Cognitive Technology, pp.190199, Washington, D.C. 1997.

[19] d' A. Flores and H. Saito, "Lexical decomposition of complex kanji characters in Japanese readers," Psychological Research, vol.55, pp.52-63, 1993.

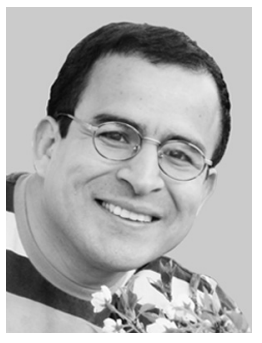

Luis Inostroza Cueva earned a B.E. from Antenor Orrego Private University in 1996 and an M.E. from The University of Tokushima in 2007. In 2005, he was awarded a scholarship from the Japanese Government Ministry of Education, Culture, Science, and Technology. Since 2008, he has been conducting $\mathrm{PhD}$ research at the Tokyo Institute of Technology. His research has been internationally recognized in academic societies, including several awards. He is an FIE New Faculty Fellow Award Recipient (2009), a Frontiers in Education grant winner (2009), and the Graduate School of Decision Science and Technology paper award winner (Tokyo Institute of Technology, 2010). Since 2009, he has been a member of the extended community of the American National Academy of Engineering (NAE), with the Center for the Advancement of Scholarship on Engineering Education (CASEE). His main research interests include (but are not restricted to) language and cognition. He is best known in cognitive science (in the field of kanji learning) for his invention of the LV Approach.

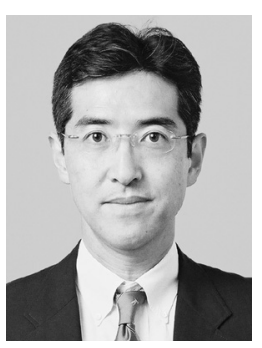

Masao Murota earned a B.E., an M.E. and a Dr. Eng. degree in electronics from the Tokyo Institute of Technology in 1986, 1988 and 1991 respectively. From 1991 to 1997 he worked for Toshiba Corporation with the Research and Development Center. Since 1997, he has been with the Tokyo Institute of Technology, where he is now an Associate Professor in the Graduate School of Decision Science and Technology. In 2007, he was a visiting researcher at Oregon State University. His main research field is educational technology, which includes developing Web-based learning systems and tools. 